\title{
$\checkmark_{\text {DOE/PC/91297. } \frac{T 4}{1}}$
}

Quarterly Technical Progress Report

(September 1, 1993 to November 30, 1993)

\section{A COMPUTATIONAL MODEL FOR \\ COAL TRANSPORT AND COMBUSTION}

Grant Number: DE-FG22-91PC91297

Goodarz Ahmadi

Department of Mechanical and Aeronautical Engineering

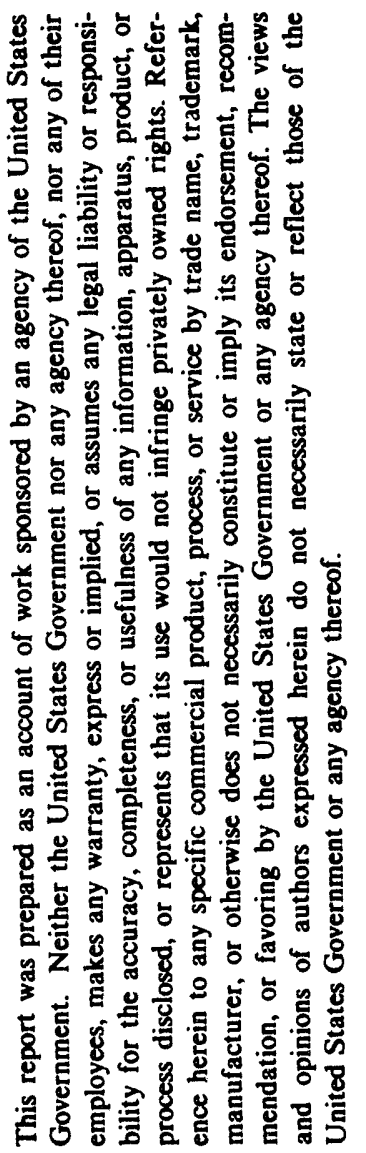

Clarkson University

Submitted to

U.S. Department of Energy

Pittsburgh Energy Technology Center

Attention:

Dr. Mehrdad Massoudi

Project Officer 


\section{A COMPUTATIONAL MODEL FOR \\ COAL TRANSP'ORT AND COMBUSTION}

Grant Number: DE-FG22-91PC91297

Project Period: September 1, 1991 to August 31, 1994

Contract Recipient: Clarkson University

Project Principal Investigator: Goodarz Ahmadi

DOE Project Officer: Dr. Mehrdad Massoudi

\section{SUMMARY}

In the period of September 1, 1993 to November 30, 1993, further progress was made of the theoretical and copmutational modeling of turbulent two-phase flows, as well as granular flows. The effect of bumpy wall on flow of granular materials was also analyzed. The special case of Couette flow was studied. Further progress was made in the experimental study of mono-layer simple shear flow device. Preliminary data concerning the shearing of $12 \mathrm{~mm}$ multi-color glass particles are obtained. The preliminary experimental data shows the expected variation.

A detailed progress report for project review was also prepared which includes the recent development. The description of this report follows. 


\section{A COMPUTATIONAL MODEL FOR \\ COAL TRANSPORT AND COMBUSTION \\ Grant Number DE-FG22-91PC91297}

Project Principal Investigator: Goodarz Ahmadi

Organization: Clarkson University

Address: Department of Mechanical and Aeronautical Engineering

Potsdam, NY 13699-5725

Telephone Number: (315) 268-2322

\section{PROJECT PERSONNEL}

Principal Investigator: Goodarz Ahmadi

Research Interests: Multiphase and Granular Flows, Turbulence Modeling, Aerosols and Computational Fluid Mechanics.

Time Devoted to the Project: 20\% During Academic Year, 45\% in Summer

Education: B.S., 1965; M.S., Purdue University, 1968; Ph.D., Purdue University, 1970.

\section{Employment History:}

(1982-Present) Professor and (1991-Present) Chairman, Department of Mechanical and Aeronautical Engineering, Clarkson University.

(1970-72) Assistant Professor, (1972-76) Associate Professor, (1976-81) Professor, Department of Mechanical Engineering, Shiraz University.

Visiting Professor/Scientist, (1981) University of Calgary, (Summer 1978) University of New Castle Upon Tyne, (Summer 1977) Northwestern University, (Summer 1976) Imperial College, (Summer 1975) Princeton University, (1974-75) University of Saskatchewan.

\section{Honors and Awards:}

"University Distinguished Teaching Award," Clarkson University (1988); "University Outstanding Advisor Award," Clarkson University (1986) and (1988); "Pi Tau Sigma Distinguished Teaching Award," Clarkson University (1984), (1986), (1988) and (1990).

Publications: Two Books and more than three hundred journal articles.

\section{Sample Relevant Recent Publications Not Emanating From This Project:}

Computer Simulation of Deposition of Aerosols in a Turbulent Channel Flow with Rough Wall, Aerosol Sci. Technology 18, 11-24 (Co-authored with A. Li) (1993).

Brownian Particles Deposition in a Directly Simulated Turbulent Channel Flow, Physics of Fluids A 5, 1427-1432 (Co-authored with H. Ounis and J.B. McLaughlin) (1993).

Experimental Investigation of Dust Particle Deposition in a Turbulent Channel Flow, J. Aerosol 
Science 24, 795-815 (Co-authored with W. Kvasnak, R.G. Bayer and M.A. Gaynes) (1993).

Deposition of Aerosols on Surfaces in a Turbulent Channel Flow, Int. J. Engng. Sci. 31, 435-451 (Co-authored with A. Li) (1993).

A Sublayer Model for Turbulent Deposition of Particles in Vertical Ducts with Smooth and Rough Surfaces, J. Aerosol Sci. 24, 45-64 (Co-authored with F. Fan) (1993).

Aerosol Particle Deposition with Electrostatic Attraction in a Turbulent Channel Flow, J. Colloid Interface Science 158, 476-482 (Co-authored with A. Li) (1993).

\section{Additional Project Personnel}

Jianfa Cao (Ph.D Student), Computational Modeling of Granular and Two-Phase Flows.

Kenneth Elliott (M.S. Student), Experimental Study of Particulate Flows.

Amy Li (Ph.D Student), Computational Modeling of Two-Phase Flows.

Sameer Abu-Zaid (Ex-Ph.D Student), Theoretical Modeling of Granular and Two-Phase Flows.

Daning Ma (Ex-Ph.D Student), Theoretical and Computational Modeling of Granular Flows.

Showkat J. Chowdhury (Ex-Ph.D Student), Theoretical and Computational Modeling of Turbulent Flows.

\section{PROJECT OVERVIEW}

Project Objectives: The objective of this project is to develop an accurate model describing turbulent flows of two-phase coal mixtures, rapid flows of granular flows including bulk coal transport and turbulent coal combustion processes. The other main objective is to develop a computational model for predicting granular and turbulent two-phase flows in various regions of industrial interest. Experimental verification of the foundation of the model is also included in the study.

Significance To Fossil Energy Program: A completely satisfactory theory describing the bulk coal transport including the interstitial fluid effects does not exisi. This is particularly the case for turbulent flows of dense coal particle-liquid mixtures and chemically active coal combustor flows. Coal-fluid mixtures and bulk transports, and operation of coal combustors accounts for a substantial portion of the cost of coal energy conversion systems. The major increase in cost arises from the need to over-design these facilities to guarantee reliability. Understanding the flow behavior of relatively dense coal slurries and bulk solids in various geometries including coal combustors, are indispensable to economical design of the needed equipment. This project aims to develop a sound practical model for coal transport and combustion. In addition, a computational predictive capability for analyzing rapid flows of granular coal particles, and turbulent flows of dense or dilute multiphase coal mixtures will be provided.

Relationship To Other DOE Funded Projects: Most other DOE funded research projects (that the PI is aware of) are concerned with the chemistry of coal and coal processing. In this regard the 
present project will be complementary to those other major efforts in that the important fluid mechanical aspects of coal transport and combustion are being addressed.

\section{Project Funding History:}

Project Period: September 1, 1991 to August 31, 1994

Funding: Grant Number DE-FG22-91PC91297, \$200,000 total DOE funding, including \$133,715 direct cost and $\$ 66,285$ indirect cost. The grant also includes $\$ 100,880$ Clarkson University Cost Sharing for student tuition and equipment.

\section{SCIENTIFIC AND TECHNICAL CONTENT}

Schedule of Major Research Activities: In the last two years many of the major objectives of this project was accomplished. These are summarized in this section.

\section{Theoretical Model Development}

i) An advanced thermodynamically consistent stress-transport model describing rapid granular flows was developed. This model contains the modern kinetic theories of granular materials as special cases.

ii) An accurate thermodynamically consistent rate-dependent model describing turbulent flows of dilute and dense two-phase flows was developed. The new model accounts for the effects of phasic fluctuation energies, their transport and interactions.

iii) An advanced thermodynamically consistent stress-transport model for turbulent flows was developed. This model contains the recent advance turbulent models as special limiting cases.

\section{Computational Model Development}

i) A computational model for analyzing rapid granular parallel flows was developed, and several examples were solved. The previously developed kinetic model for granular flow which includes frictional losses was used in these analysis.

ii) A computational model for analyzing rapid granular flows in complex geometry of industrial interest was developed. Examples of three-dimensional particulate flows in an obstructed duct, as well as gravity flow done an incline chute were solved. The previously developed kinetic model for granular flow was used in these analysis.

iii) A computational model for analyzing turbulent two-phase parallel flows is being developed. The thermodynamically consistent model developed earlier is used in this analysis. The preliminary results are quite encouraging.

iv) A computational model for analyzing two-phase turbulent flows in complex geometry of industrial interest is being developed. The previously developed thermodynamically consistent model is used in this computational model. 


\section{Experimental Study}

i) A shear flow device for obtaining experimental data on rapid shearing of granular material was constructed. The objective is to provide detailed experimental data for verify the foundation of the new models. The preliminary results are quite encouraging.

Scientific and Technical Issues: Currently the following issues are being addressed:

\section{Fundamental Issues}

i) The boundary conditions have significant effects on the nature of rapid granular and turbulent two-phase flows. We are trying to understand the nature of boundary conditions suitable for analyzing granular and two-phase flows. While the boundary conditions for idealized granular flows was, in part, studied in the literature, developing suitable boundary conditions for dense two-phase turbulent flows has never been properly addressed.

ii) The effect of chemical reactions needs to be added to the thermodynamically consistent models for rapid granular and turbulent flows. This will enable the model to become suitable for application to combustor flows.

iii) The material parameters need to be evaluated for granular coal and coal-fluid mixtures. The entire range of dilute and dense two-phase flows must be considered. The nonsphericity of coal particles and their effects need to be addressed.

\section{Computational Model Issues}

i) Realistic (bumpy and/or frictional) boundary conditions need to be included in the computational models for analyzing granular and two-phase flows.

ii) The computational models for analyzing turbulent two-phase flows in complex geometry of industrial interest must be developed and validated.

iii) Practical examples of dense and dilute two-phase turbulent flows must be solved.

\section{Experimental Issues}

i) Comprehensive experimental data for mean velocity, fluctuation energy and solid volume profiles must be obtained.

Approach Taken: As noted before a comprehensive study encompusing theoretical formulation, computational modeling and experimental investigation is being conducted to provide insight into the problem of bulk solid transport, as well as two-phase turbulent flows.

Importance of the Research: This project is expected to provide a detail understanding of granular and two-phase flow processes. In addition, a computational predictive capability for handling practical industrial problems will be provided. These tool should enable the engineer to improve the efficiency of their coal handling equipment, and to improve their designs. 


\section{PROJECT OUTPUT}

\section{Highlight of Recent Accomplishments}

\section{A THERMODYNAMICALLY CONSISTENT RATE-DEPENDENT MODEL FOR TURBULENT TWO-PHASE FLOWS}

A thermodynamically consistent theory for dense two-phase flow was developed. The new model includes the transport equations for the phasic fluctuation kinetic energies. Anisotropic and rate-dependent expressions for the phasic stress tensors were derived. For dense solid-liquid mixtures, the material parameters of the model were evaluated from the limiting conditions. The special case of simple shear flows of glass beads-water mixtures were studied. The coefficient of restitution for glass bead collision in water was experimentally measured and used in the model predictions.

Figure 1 shows the predicted shear and second normal stresses of the present model along with the data of Hanes and Inman for glass beads of $1.1 \mathrm{~mm}$ diameter in water mixture. The dashed lines are the predicted particulate and fluid phasic stresses. It is observed that particulate phase contributions to the shear and normal stresses increase as solid volume fraction increases. Reasonable agreement is observed between the experimental data and the predictions of the present model.
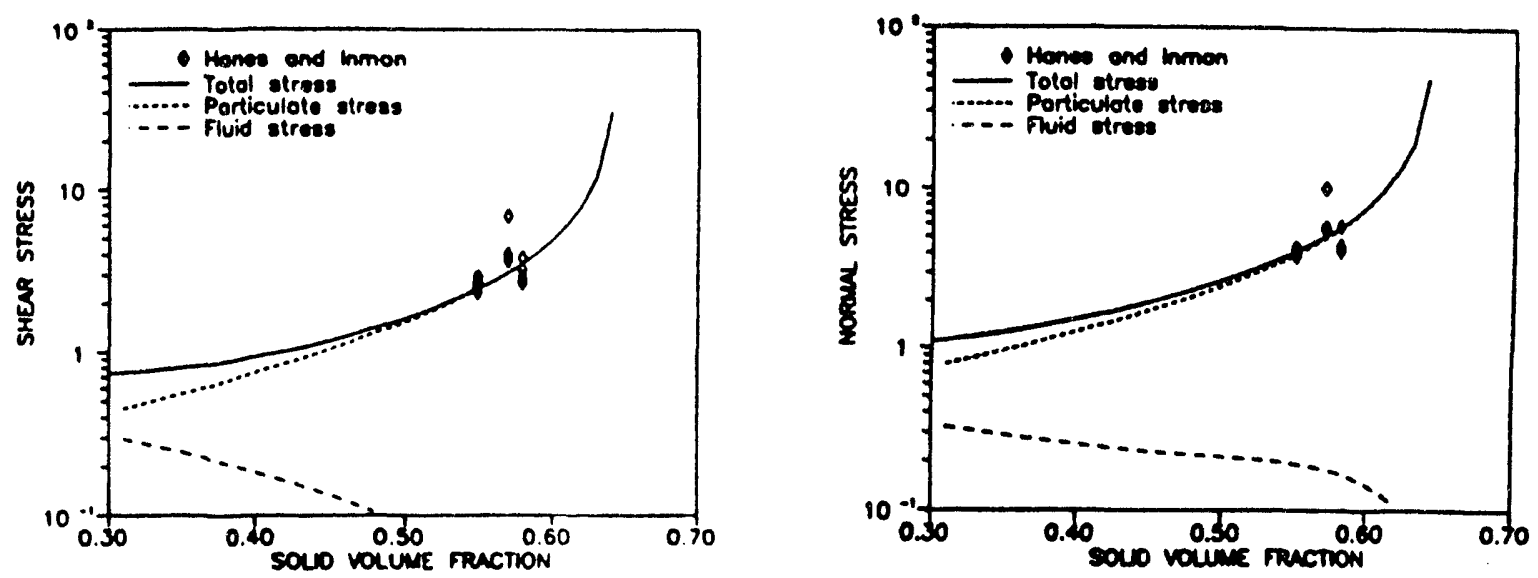

Figure 1. Variations of total and phasic shear and normal stresses with solid volume fraction.

Figure 2 shows variations of shear stress with shear rate for two values of solid volume fraction along with the experimentally measured stresses of Hanes and Inman for $1.1 \mathrm{~mm}$ glass beads in water. Although some deviations are observed, the predicted stresses are in good agreement with the experimental data.

Unlike the available models, the new rate-dependent formulation is capable of predicting the production and dissipation rates of phasic fluctuation energies. Figure 2 shows variations of nondimensional phasic fluctuation energy production with solid volume fraction for a mixture of $1.1 \mathrm{~mm}$ glass beads in water. This figure shows that for large solid volume fractions, the fluctuation energy is mainly produced by the particulate phase and the contribution of the fluid phase is negligible as $v$ approaches the limiting maximum solid volume fraction of about 0.64 . For smaller values of $v$, the fluid phasic energy production become rather large and that of the solid phase decreases. Additional details of the model were described in two technical reports, copies of 
which are enclosed.
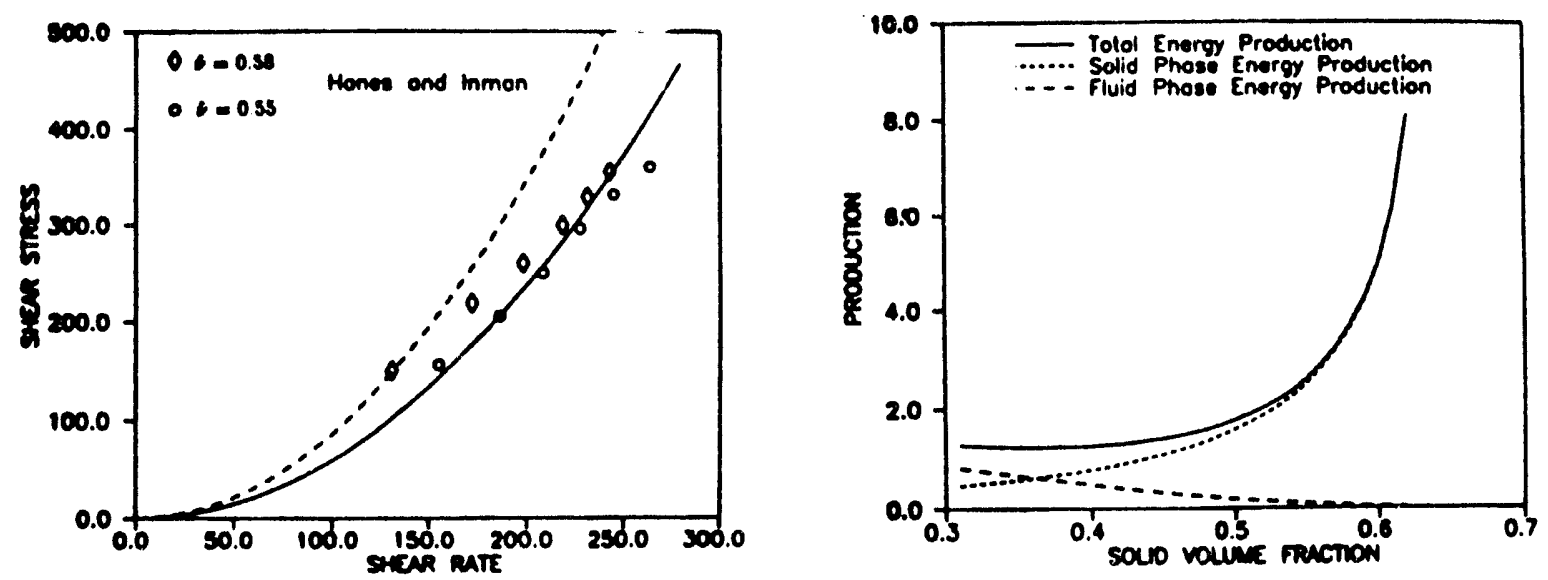

Figure 2. Variations of shear stress with shear rate and the phasic fluctuation energy production with solid volume fraction for $1.1 \mathrm{~mm}$ glass beads in water.

\section{A THERMODYNAMICALLY CONSISTEINT STRESS TRANSPORT MODEL FOR RAPID GRANULAR FLOWS}

The basic conservation laws governing the flow of rapid granular materials in a rotating frame of reference along with a transport equation for the granular kinetic stress tensor and the corresponding Clausius-Duhem inequality were considered. The thermodynamic of the mean granular motion was described and based on the averaged entropy inequality, constitutive equations for the pressure-strain, collisional stress, and flux of granular stress tensors were formulated. The model parameters for the special case of spherical nearly elastic particles were evaluated. The resulting stress transport model was used and the case of rapid simple shear flow of a collection of particles was analyzed. The preliminary results for the granular stresses and the normal stress differences were compared with the available simulation data and good agreement was observed.
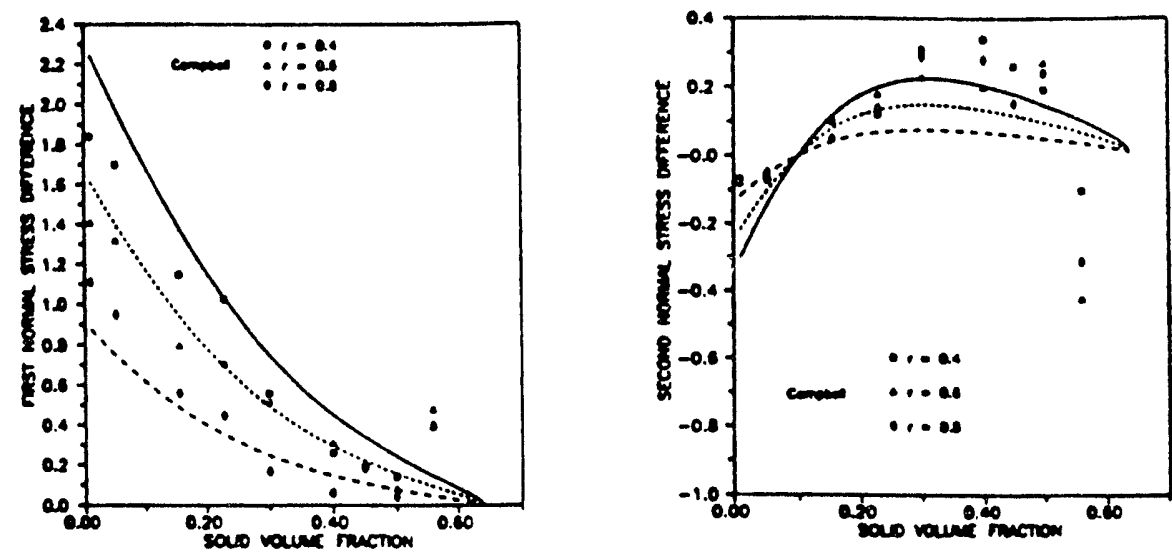

Figure 3. Variations of nondimensional first and second normal stress differences with solid volume fraction.

Figure 3 shows variations of first and second normal stresses with solid volume fraction for different values of coefficient of restitution. The simulation results of Campbell is also shown in this figure for comparison. While some deviations are observed, the predicted normal stress differences are in good agreement with the simulation results. 


\section{ANALYSIS OF RAPID GRANULAR FLOWS INCLUDING FRICTIONAL LOSSES}

The kinetic model for particulate flows, including frictional energy losses, was used to analyze the rapid granular flows in a Couette geometry, gravity flows down an inclined chute and a vertical channel. The equations of motion were solved using a fourth-order Runge-Kutta numerical scheme. The corresponding mean velocity and solid volume fraction profiles for the flow in a vertical channel are shown in figure 4. The data of Savage (1979) is also shown in this figure for comparison. It is observed that the predicted roughly paraboidal mean velocity profile is in good agreement with data of Savage. Figure 4 also shows that the fluctuation energy has a rather high value in the shearing region near the wall, and decreases to its minimum value near the centerline. The solid volume fraction has an opposite trend of variation. It has a somewhat low value near the wall, and increases to its peak value near the centerline.

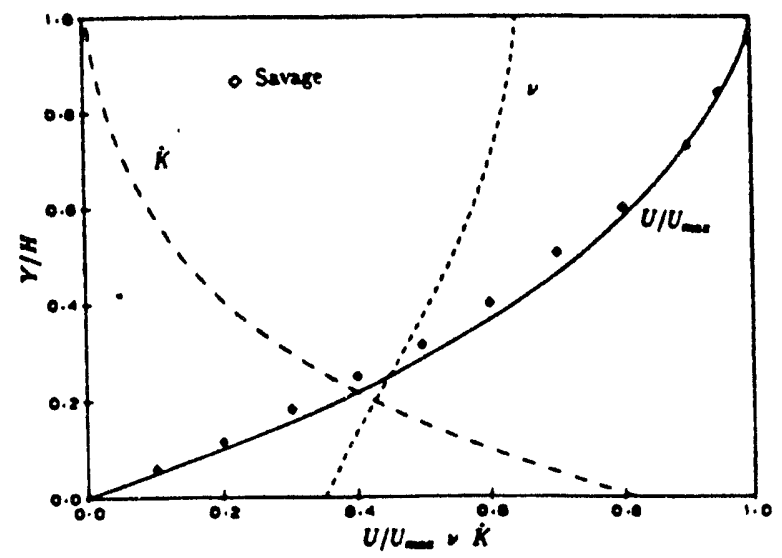

Figure 4. Variations of mean velocity, fluctuation energy, and solid volume fraction profiles for granular flows down a vertical channel.

\section{GRANULAR FLOWS AROUND A BLOCK}

A computational model for analyzing granular flows in non-simple regions was developed. The special case of granular flow around a rectangular cylindrical block in a channel was studied in details. The details of the computational model and the discretization used were described in a published report, a copy of which is enclosed.

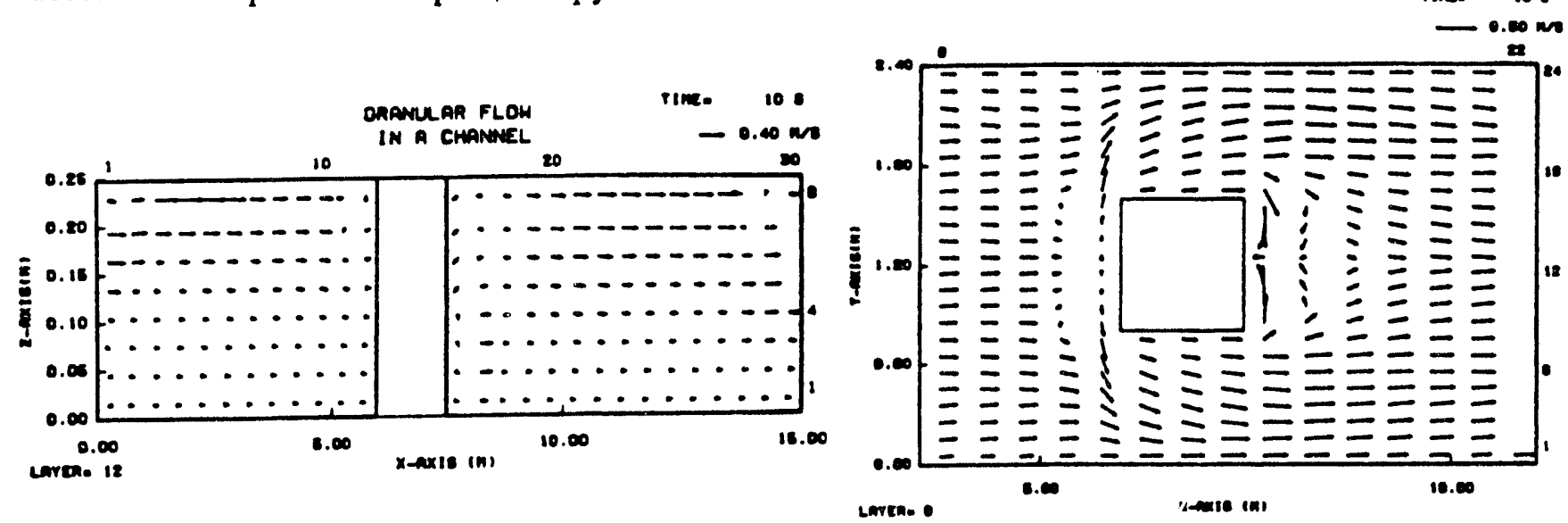

Figure 5. Velocity vector plot at the surface layer and plane of symmetry at $t=10 \mathrm{sec}$.

Sample computer simulation results for the velocity conditions are shown in Figure 5. It 
is observed that the block significantly distorts the flow field. This figure shows that a source of granular materials seems to appear at the center line in front of the block which interacts with the incoming uniform velocity field. A reverse flow region forms in front of the block which penetrates the upstream flow field up to about one meter. On both sides of the block, the flow accelerates and a separation bubble is formed behind the block. Further downstream, the wake region can be seen. This figure also shows that the granular particles move upward in front of the block, while they move downward behind the block. These lead to the formation of source and sink type flows in front and behind the block noticed at the surface layer.

\section{GRANULAR FLOWS WITH BUMPY BOUNDARY}

The interactions of particle with the boundary is known to significantly affect flow and transport of granular materials. The schematics of a bumpy boundary is shown in figure 6 . The kinetic model of granular materials including frictional losses was used in the analysis. The presence of bumpy boundary conditions leads to a strongly couple system of governing equations which has to be solve numerically even for simple case of Couette flow. A special discretization scheme was developed and the mean velocity, the fluctuation kinetic energy and the solid volume fraction profiles for granular flows between two parallel was were evaluated. The results for different values of friction coefficients are plotted in figure 6 . The molecular dynamic simulation results of Savage and Dai (1992) for the frictionless particles are also shown in this figure for comparison. It is observed that the model predictions are in excellent agreement with the molecular dynamic simulation results.
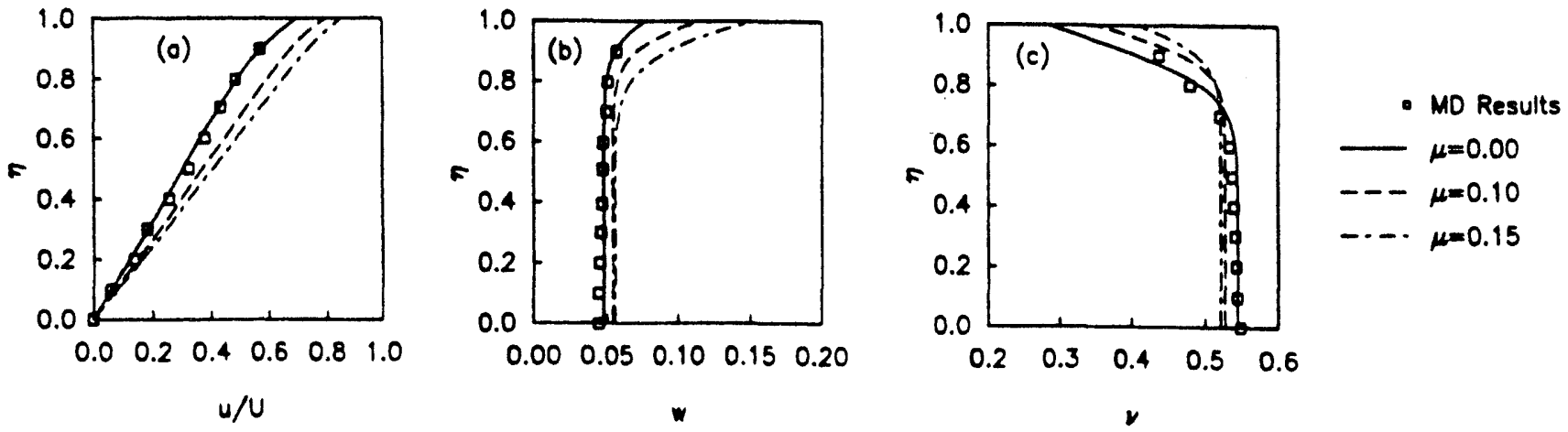

Figure 6. Schematics of a bumpy wall and variations of mean velocity, fluctuation energy and solid volume fraction profiles. Comparison with the molecular dynamic simulation of Savage and Dai (1992).

Figure 6 shows that there are a significant amount of slip at the wall. The slip velocity decreases as the friction coefficient increases. It is also observed that the fluctuation velocity is rather large near the wall and approaches a constant away from the wall. The solid volume fraction, on the other hand, is small near the wall and increases with distance from the wall. Figure 6 also shows that $v$ is roughly constant in the core region. These results show that the commonly used assumptions of a linear mean velocity and constants values of kinetic energy and solid volume fraction for Couette flow of granular materials are not correct.

Figure 7 shows Gravity flows the model prediction results for granular gravity flow over a bumpy incline as compared with the experimental data of Johnson et al. (1990). In these computations, the earlier theory of Abu-Zaid and Ahmadi(1990) for inelastic and frictional particle were used. The agreement of these model predictions with the data is quite encouraging. 

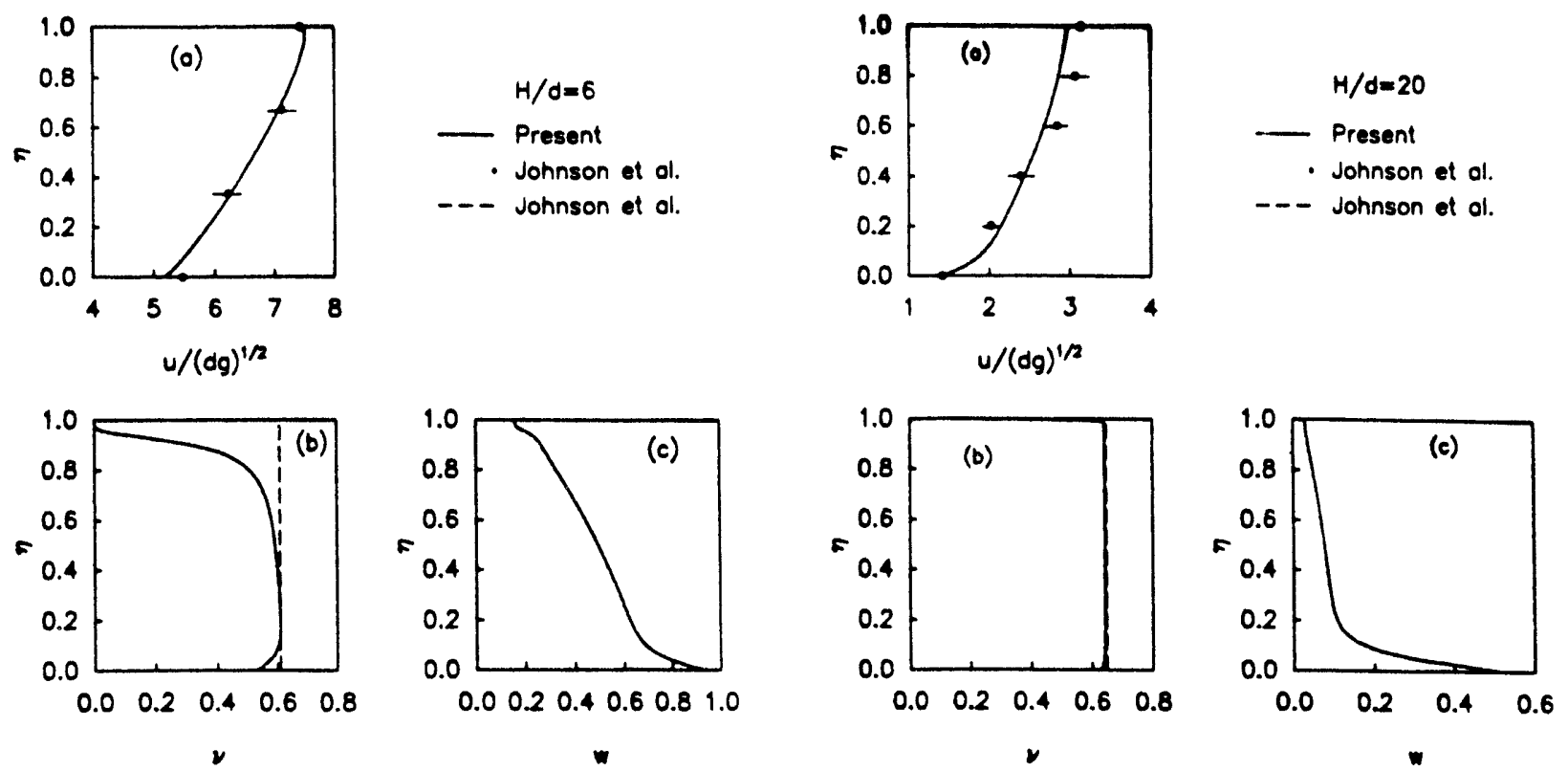

Figure 7. Sample prediction results for granular over a bumpy incline.

Comparison with the experimental data of Johnson et al. (1990).

\section{TWO-PHASE FLOWS}

Progress has been made in formulation of appropriate equations for motion of dense two-phase mixtures, and the development of the computational scheme of solution. Effort has been made to include the effects of fluid turbulence, particles fluctuation energy and collisional stresses into the computational model. The suitability of different boundary conditions for the fluid phase and particulate phase are also studied. Figure 10 shows sample preliminary computational results for duct flow of two-phase mixtures and comparison with the experimental data of Tsuji et al. (1984). The model predictions appear to be in reasonable agreement with the data of Tsuji et al.
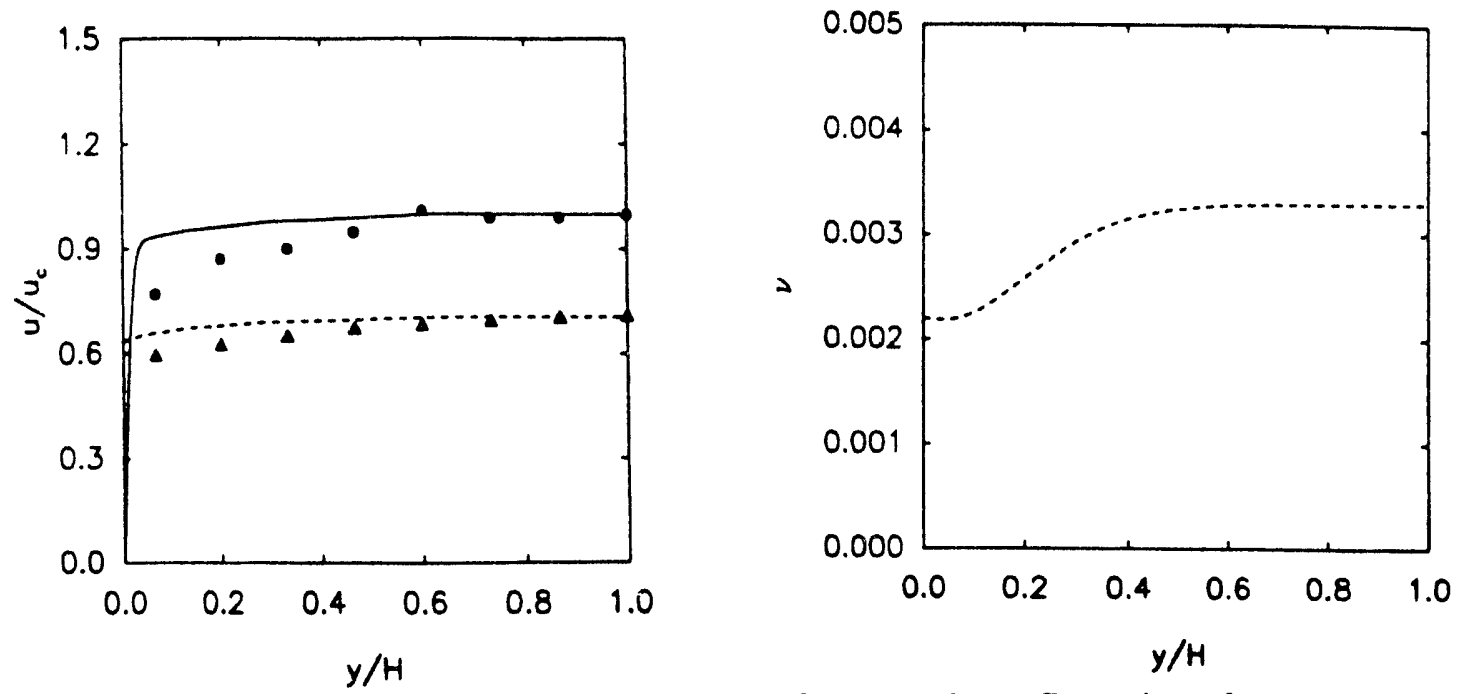

Figure 8. Sample prediction results for two-phase flows in a duct.

Comparison with the experimental data of Tsuji et al. (1984).

\section{EXPERIMENTAL STUDY}

A mono-granular simple shear flow setup was constructed and was used for the 
experimental study. Figures 9 shows the schematic of the design and a picture of the manufactured device and the shearing cell. Here multi-colored spherical glass balls which are 12 $\mathrm{mm}$ in diameter were used as granular particles. The position of the balls in consecutive frames taken 1/30th of a second apart from the test run at a shear rate of $62.831 / \mathrm{sec}$ were measured. Using this technique, the velocity vector of each particle can be calculated. The shearing region was divided into 10-15 equivalent horizontal segments. Averaging procedures are used to provide the experimental velocity and concentration profiles.
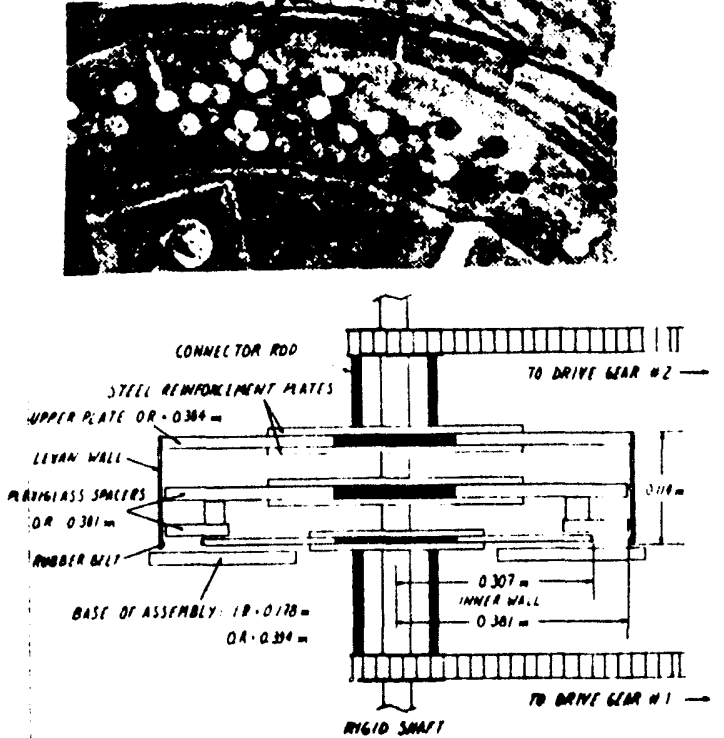

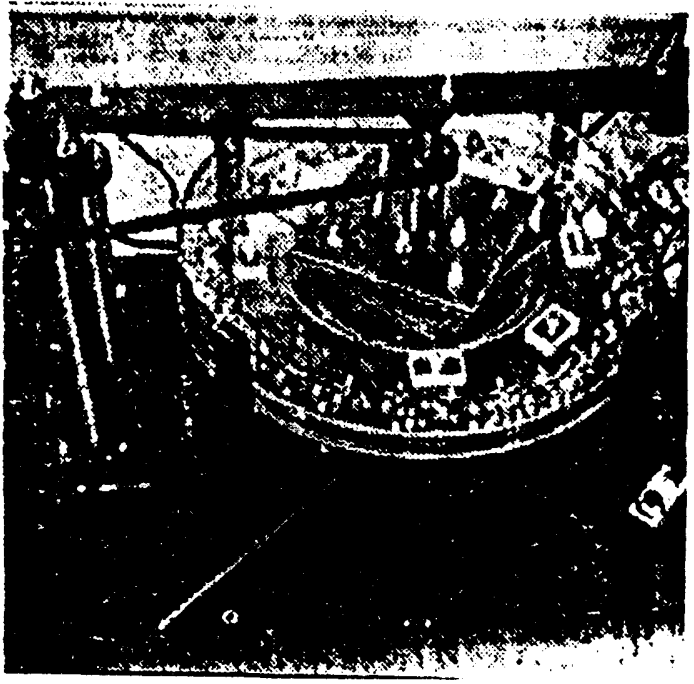

Figure 9. The schematic and a picture of the simple shear flow device.

Figui , 10 shows sample experimental data. These include mean velocity, concentration and fluctuation velocity profiles. It is observed that the significant amount of slip exist. The slip is much larger at the inner wall when compared with that of the outer wall. It appears that the centrifugal force is important for this rotating Couette flow geometry. At the inner wall, the centrifugal force moves the particle away from the wall, reduces the normal force and, as a result, the slip velocity increases. At the outer wall, however, the particles are pushed toward the wall by the centrifugal force and the slip velocity decreases. Figure 10 also shows that the axial velocity is roughly linear in the central part of the shearing region. It is observed that the solid volume fraction and fluctuation energy varies significantly across the section.
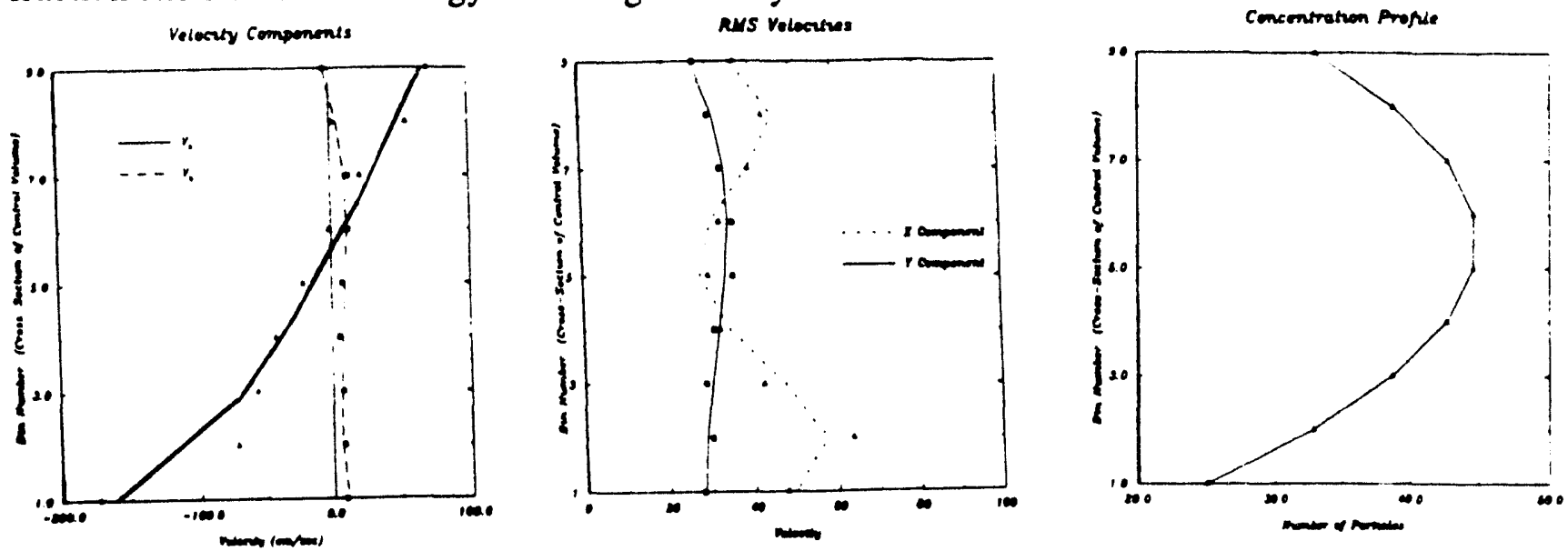

Figure 10. Preliminary experimental data for the mean velocity, solid volume fraction and fluctuation energy profiles. 


\section{Bibliography of Publications Emanating From This Project}

\section{Journals Publications}

G. Ahmadi and S.J. Chowdhury, "A Rate-Dependent Algebraic Stress Model for Turbulence," Applied Math. Modelling 15, 516-524 (1991).

H. Ounis and G. Ahmadi, "Motions of Small Particles in a Turbulent Simple Shear Flow Field Under Microgravity Condition," Physics of Fluids A 3, 2559-2570 (1991).

S. Abu-Zaid and G. Ahmadi, "A Thermodynamically Consistent Stress Transport Model for Rotating Turbulent Flows," Geophys. Astrophys. Fluid Dynamics 61, 109-121 (1991).

G. Ahmadi, "A Thermedynamically Consistent Rate-Dependent Model for Turbulence, Part I Formulation," Int. I. Non-Linear Mech. 26, 595-607 (1991).

K.A. Elrais, W. Eckerle, G. Ahmadi and A.H. Eraslan, "Simulation of Transient Three-Dimensional Natural Convection and Saturated Pool Boiling, Int. J. Numerical Methods Heat Fluid Flow 2, 39-154 (1992).

S.J. Chowdhury and G. Ahmadi, "A Thermodynamically Consistent Rate-Dependent Model for Turbulence, Part II - Numerical Results," Int. J. Non-Linear Mech. 27, 705-718 (1992).

W.G. Paff and G. Ahmadi, "On Convergence of Karhunen-Loeve Series Expansion for a Brownian Particle," J. Appl. Mech. Trans. ASME (ln Press).

S. Abu-Zaid and G. Ahmadi, "A Stress Transport Model for Granular Flows in a Rotating Frame, Int. J. Engng. Sci. 30, 1483-1495 (1992).

S.J. Chowdhury and G. Ahmadi, "Analysis of A Mixing Layer by a Rate-Dependent Turbulence Model," ASCE, Journal of Engineering Mechanics 119, 1700-1706 (1993).

D. Ma, A.H. Eraslan and G. Ahmadi, "A Computer Code for Analyzing Transient Three-Dimensional Rapid Granular Flows in Complex Geometries, Computer Fluids 22, 25-50 (1993).

S. Abu-Zaid and G. Ahmadi, "Analysis of Rapid Shear Flows of Granular Materials by a Kinetic Model Including Frictional Losses," Powder Technology (In Press).

S. Abu-Zaid and G. Ahmadi, A Thermodynamically Consistent Rate-Dependent Model for Turbulent Two-Phase Flows, Part I - Formulation," MAE Report-281 November (1993). Also submitted to Int. J. Multiphase Flows.

S. Abu-Zaid and G. Ahmadi, "A Thermodynamically Consistent Rate-Dependent Model for Turbulent Two-Phase Flows, Part II - Simple Shear Flows," MAE Report-282 November (1993). Also submitted to Int. J. Multiphase Flows. 


\section{Conference Presentations}

G. Ahmadi and S. Abu-Zaid, "Overview of Thermodynamical Approach to Modeling Turbulent Flows of Two-Phase Solid-Liquid Mixtures," International Conference on Multiphase Flow '91-Tsukuba, Tsukuba, Japan, September 24-27, 1991.

G. Ahmadi and S. Abu-Zaid, "A Thermodynamically Consistent Model for Turbulent Two-Phase Flows," 28th Annual Technical Meeting of the Society of Engineering Science, Gainsville, FL, November 6-8, 1991.

G. Ahmadi and S. Abu-Zaid, "Analysis of Rapid Shear Flows of Granular Materials by a Stress Transport Model," 28th Annual Technical Meeting of the Society of Engineering Science, Gainsville, FL, November 6-8, 1991.

G. Ahmadi, "Overview of Thermodynamical Modeling of Turbulent Flows of Multiphase Mixtures," Proceedings of the International Conference on Engineering Application of Mechanics, Vol. 3, Sharif University of Technology, Ed. by M.S. Sadeghipour et al., June 9-12, 1992, 386-399.

G. Ahmadi and S. Abu-Zaid, "A Thermodynamically Consistent Stress Transport Model for Rapid Granular Flows," The 23nd Annual Meeting of the Fine Particle Society, Las Vegas, Nevada, July 13-17, 1992.

G. Ahmadi and S. Abu-Zaid, "A Model for Turbulent Two-Phase Flows," MEET'N'93, ASME/ASCE/SES Joint Meeting, University of Virginia, Charlottsville, VA, June 6-9, 1993.

G. Ahmadi, D. Ma, and A. Eraslan "Rapid Granular Flow in Complex Geometry Regions," .MEET'N'93, ASME/ASCE/SES Joint Meeting, University of Virginia, Charlottsville, VA, June 6-9, 1993.

G. Ahmadi, "A Computational Model for Coal Transport and Combustion," University Coal Research Contractors" Review Conference, Pittsburgh, PA, June 23-25, 1993.

G. Ahmadi and S. Abu-Zaid, "Turbulent Two-Phase Flow of Dense Mixtures-A Thermodynamically Consistent Model," The 24th Annual Meeting of the Fine Particle Society and First International Conference on Pharmaceutical Sciences and Technology, Chicago, Illinois, August 23-28, 1993.

J. Cao, and G. Ahmadi, "Numerical Simulation of Granular Couette Flows Between Two Rough Parallel Plates," The 24th Annual Meeting of the Fine Particle Society and First International Conference on Pharmaceutical Sciences and Technology, Chicago, Illinois, August 23-28, 1993.

G. Ahmadi, D. Ma, and A. Eraslan "Analysis of Transient Rapid Granular Flows in a Channel with an Obstruction," The 24th Annual Meeting of the Fine Particle Society and First International Conference on Pharmaceutical Sciences and Technology, Chicago, Illinois, August 23-28, 1993. 

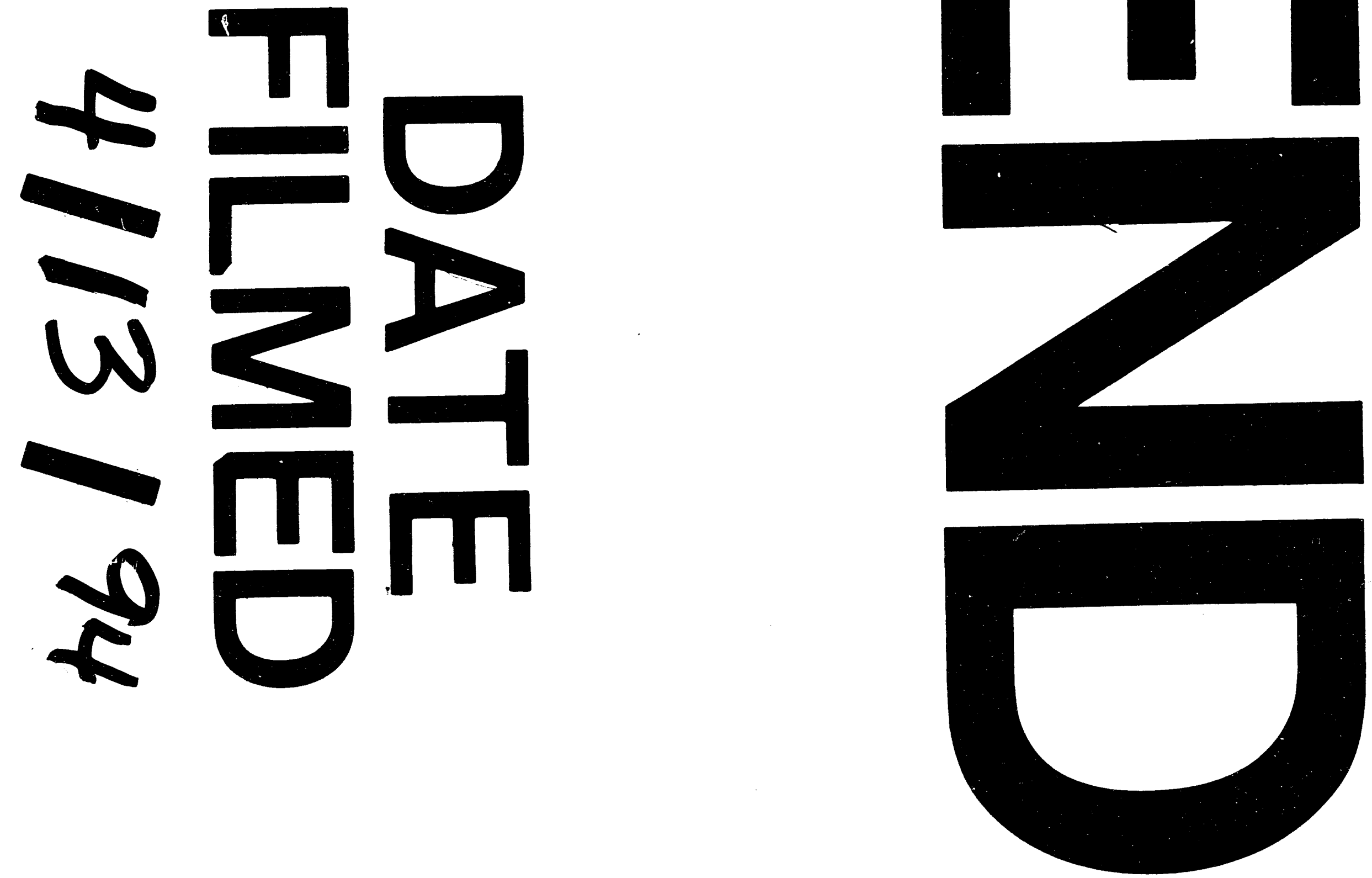


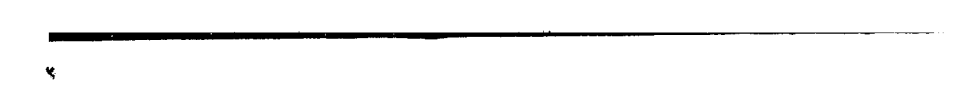

